\title{
PULMONARY ARTERY ANEURYSMS IN BEHCET'S DISEASE: A CASE REPORT
}

Juliana Almeida Vieira1,^, Flávia Nogueira Chiva ${ }^{1}$, Paula Bergamasco da Silva ${ }^{1}$ Elza Maria Gouvea Avila², Lúcia Stella Seiffert Assis Goulart ${ }^{1}$ Virginia Moça Trevisani ${ }^{1}$

1.Universidade de Santo Amaro, São Paulo (SP), Brazil. 2.Hospital São Luiz, São Paulo (SP), Brazil.

*Corresponding author: juliana.vieira1994@gmail.com

\section{BACKGROUD}

Behçet's disease (BD) is a chronic, recurrent, multisystemic vasculitis whose etiopathogenesis is not completely known yet. To be classified as having the disease, patients must score 4 points according to the 14th International Criteria for Behçet's Disease (ICBD). Although vascular impairment in the disease occurs in 25 to $30 \%$ of cases, this is the largest cause of disease-related mortality. Behçet's disease is the leading cause of pulmonary artery aneurysms, which can occur in major segments or segmental branches, indicating poor prognosis. We present the case of a patient diagnosed with BD with pulmonary artery aneurysms. The patient gave consent for this publication.

\section{CASE REPORT}

Male, 34 years old, admitted for dyspnea and ventilator-dependent thoracic pain for 3 months associated with productive cough, intermittent fever and prostration. At admission, he was in a bad general state, sweating, acyanotic, dyspneic, Glasgow 14 . Pulmonary auscultation with vesicular murmur present and crackling in the left lower third. He had antecedent of deep venous thrombosis in the left inferior limb in irregular use of warfarin. Two pairs of blood cultures and urine culture were negative, renal and liver function preserved, elevated leucocytes and PCR. Negative P-ANCA, C-ANCA, anticardiolipin antibodies and lupus anticoagulant. Serologies for viral hepatitis and HIV negative. Contrast-enhanced chest computed tomography (CT) was performed showing pulmonary thromboembolism (PESI III) with occlusion of the right posterior basal pulmonary artery and sparse aneurysms in the pulmonary arteries, most of them sacculiform reaching $4.4 \mathrm{~cm}$ and presenting with parietal thickening/laminar eccentric thrombus. Full anticoagulation with enoxaparin and antibiotic therapy for pulmonary focus were initiated. During hospitalization, he had developed erythema nodosum and oral ulcers. He also had a previous episode of genital ulcers. Negative pathergy test. At discharge, he was referred to a rheumatology clinic using colchicine and rivaroxaban, where he started pulse therapy with methylprednisolone and after cyclophosphamide for 6 months. After the 4 th cycle, he underwent a new contrast CT that showed sparse pulmonary aneurysms in branches of the pulmonary artery bilaterally, mostly sacculiform and with parietal thickening/eccentric thrombus, the largest in the upper left lobar artery $(7.7 \times 7.5 \mathrm{~cm})$. Patient reported clinical improvement, despite radiological worsening.

\section{CONCLUSION}

Detailed investigation for accurate BD diagnoses is very important. The basic treatment for BD patients with aneurysmal lesions is glucocorticoids and cyclophosphamide, but refractory cases can occur; then we must consider anti-TNF-a therapy or surgical and interventional procedures. 